\title{
Core-collapse and Type la supernovae with the SKA
}

\author{
M.A. Pérez-Torres ${ }^{* 1,2,3}$, A. Alberdi ${ }^{1}$, R. J. Beswick ${ }^{4}$, P. Lundqvist ${ }^{5}$, R. Herrero-Illana ${ }^{1}$, \\ C. Romero-Cañizales ${ }^{6,7}$, S. Ryder ${ }^{8}$, M. della Valle ${ }^{9}$, J. Conway ${ }^{10}$, J.M. Marcaide ${ }^{11,12}$, \\ S. Mattila ${ }^{13}$, T. Murphy ${ }^{14}$, E. Ros ${ }^{15,11,16}$
}

${ }^{1}$ Instituto de Astrofísica de Andalucía (IAA-CSIC), E-18008 Granada, Spain

${ }^{2}$ Centro de Estudios de la Física del Cosmos de Aragón, E-44001 Teruel, Spain

${ }^{3}$ Depto. de Física Teórica, Facultad de Ciencias, Universidad de Zaragoza, E-50009, Spain

${ }^{4}$ Jodrell Bank Centre for Astrophysics/e-MERLIN, The University of Manchester, M13 9PL, UK

${ }^{5}$ Stockholms Observatorium, Sweden

${ }^{6}$ Instituto de Astrofísica, Pontificia Universidad Católica de Chile, Macul, Santiago, Chile

${ }^{7}$ Millennium Institute of Astrophysics, Vicuña Mackenna 4860, 7820436 Macul, Santiago, Chile

${ }^{8}$ Australian Astronomical Observatory, P.O. Box 915 North Ryde NSW 1670, Australia

${ }^{9}$ Osservatorio Astronomico di Capodimonte - INAF, 80131 Napoli, Italy

${ }^{10}$ Onsala Space Observatory, Chalmers, Tekniska Högskola, SE-439 92 Onsala, Sweden

${ }^{11}$ Dept. Astronomia i Astrofisica, Universitat de València, E-46100 Burjassot, Spain

${ }^{12}$ DIPC, Paseo Manuel Lardizabal, Donostia-San Sebastián, Spain

${ }^{13}$ Finnish Centre for Astronomy with ESO (FINCA), University of Turku, FI-20014, Finland

${ }^{14}$ The University of Sydney, 44 Rosehill Street, NSW 2006, Australia

${ }^{15}$ Max-Planck-Institut für Radioastronomie, Auf dem Hügel 69, D-53121 Bonn, Germany

${ }^{16}$ Observatori Astronòmic, Universitat de València, E-46980 Paterna, Valéncia, Spain

E-mail: torreseiaa.es

Core-collapse SNe (CCSNe): Systematic searches of radio emission from CCSNe are still lacking, and only targeted searches of radio emission from just some of the optically discovered CCSNe in the local universe have been carried out. Optical searches miss a significant fraction of CCSNe due to dust obscuration; CCSN radio searches are thus more promising for yielding the complete, unobscured star-formation rates in the local universe. The SKA yields the possibility to piggyback for free in this area of research by carrying out commensal, wide-field, blind transient survey observations. SKA1-SUR should be able to discover several hundreds of CCSNe in just one year, compared to about a dozen CCSNe that the VLASS would be able to detect in one year, at most. SKA, with an expected sensitivity ten times that of SKA1, is expected to detect CCSNe in the local Universe by the thousands. Therefore, commensal SKA observations could easily result in an essentially complete census of all CCSNe in the local universe, thus yielding an accurate determination of the volumetric CCSN rate. Type Ia SNe: We advocate for the use of the SKA to search for the putative prompt ( first few days after the explosion) radio emission of any nearby type Ia SN, via target-of-opportunity observations. The huge improvement in sensitivity of the SKA with respect to its predecessors will allow to unambiguously discern which progenitor scenario (single-degenerate vs. double-degenerate) applies to them.

Advancing Astrophysics with the Square Kilometre Array,

June 8-13, 2014

Giardini Naxos, Sicily, Italy 


\section{Why CCSN searches in the radio?}

The limited sensitivity of pre-eMERLIN/VLA interferometric arrays has biased past radio observations of CCSNe towards the brightest events, preventing any systematic radio follow-up of CCSNe of all types. The exception has been that of Type Ib/c SNe, which due to their GRB link have been the subject of a systematic monitoring with the VLA (see, e.g., Bietenholz et al. 2014; Soderberg et al. 2006). All this makes the currently existing radio observations of CCSNe of rather limited use

In this chapter, we argue that a commensal, wide-field, transient survey with the SKA could potentially allow us to obtain a complete census of CCSNe in the local universe, and therefore will permit us to determine the true CCSN rate and thus the star-formation rate of the population of massive stars in the local universe. In addition, some specific, relevant questions that will be tackled by those observations include the following:

- Obtaining an accurate estimate of the true volumetric CCSN rate in the local universe, $\Re$. Wide-field SKA observations covering a significant area of the sky will discover many CC$\mathrm{SNe}$ in the nearby universe, and therefore will allow us to determine this relevant parameter (see Fig. 1).

- Probing the SN-CSM interaction for all CCSN types, from the relatively faint Type IIP to the extremely radio bright Type IIn SNe (Fig.2). Probing the SN-CSM interaction for all CCSN types will allow us to obtain basic, crucial information to characterize their progenitors, including mass-loss rates and, for synchrotron-self-absorbed SNe, the shock radius and the magnetic field-directly from the light curves (see, e.g., Chevalier 1998).

- Bridging the gap between Type Ibc SNe and (long) $\gamma$-ray bursts. Type Ibc are arguably the CCSNe that show the highest blastwave speeds, yet most of them are energetically much less powerful than GRBs. Recently, however, cases like SN 2009bb, with $\beta \sim 0.9$ and energy $\sim$ $10^{49} \mathrm{erg}$ seem to be intermediate cases. These "engine-driven" CCSNe could be detected with the high-sensitivity offered by the SKA, thus filling this gap in the energy-blastwave velocity parameter space of SNe-GRBs (see, e.g., Gal-Yam et al. (2006) and references therein).

- Typing CCSNe from their radio behaviour. A systematic monitoring could permit us to type CCSNe from their radio emitting properties. This is crucial for the study of the hidden SN population in (Ultra)Luminous Infra-Red Galaxies, where a spectroscopical classification, or even an optical discovery, is essentially impossible (see, e.g., Mattila et al. (2012) and Fig.1).

- Unveiling the hidden CCSN population. Horiuchi et al. (2011) found out an apparent mismatch between the measured CCSN rate (mostly from optical observations) and the cosmic massive star formation rate, which was twice as large as the measured one. However, Mannucci et al. (2007) and Mattila et al. (2012) have shown that a significant fraction of the exploding CCSNe in the local universe are hidden behind dust, and that this fraction seems to increase significantly as one goes back in the history of the universe (see Fig. 1, left panel),

\footnotetext{
* Speaker.
} 

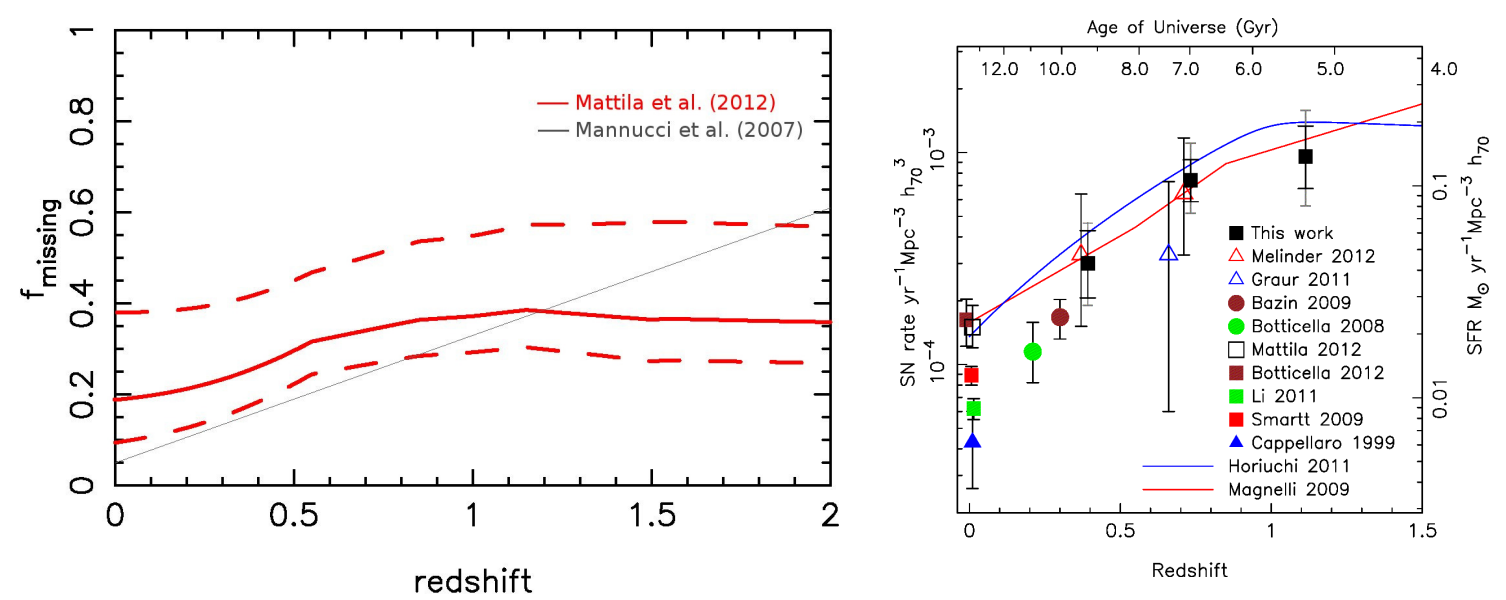

Figure 1: Left: Fraction of CCSNe missed by rest-frame optical searches as a function of redshift. The red lines show the best estimate (solid line) together with the upper and lower bounds of the missing fraction as dashed lines. The solid black line corresponds to the missing fraction from Mannucci et al. (2007). (Figure from Mattila et al. 2012.) Right: Core-Collapse Supernova rate as a function of redshift. (Figure from Dahlen et al. 2012.) The use of the SKA as a CCSN discovery machine willl reduce the relatively large uncertainty in the missing fraction of CCSNe in the local universe (left figure), as well as in the volumetric CCSN rate (right figure).

i.e., there seems to be no mismatch when the hidden CCSNe are taken into account. The results obtained by Mattila et al. (2012) were mostly based on high spatial resolution near-IR and radio observations of the local LIRG Arp 299, and showed that the combination of nearIR and radio observations with high angular resolution are very useful to study CCSNe in LIRGs (e.g. Kankare et al. 2014; Romero-Cañizales et al. 2014). Radio observations have the advantage over both optical and near-IR that the emission from CCSNe is not hampered by dust, and thus offers an excellent opportunity to determine the true core-collapse supernova rate in the local universe. In fact, most of the the CCSNe that explode in the compact, central regions of luminous and ultra-luminous infrared galaxies can essentially be found only at radio wavelengths, e.g. SN 2000ft in NGC 7469 (Alberdi et al. 2006; Colina et al. 2001), or the many CCSNe unveiled by radio observations in Arp 299A (Bondi et al. 2012; Pérez-Torres et al. 2009a), Arp 299B (Romero-Cañizales et al. 2011), or Arp 220 (Batejat et al. 2011; Parra et al. 2007).

- Correlating optical and radio properties. The combined use of optical information for both SN and host galaxy, together with the obtained peak (radio) luminosities will allow us to check whether there is a correlation between the optical and radio properties of CCSNe, as well as with their host galaxies. This will be possible by, e.g., making a combined, commensal, use of wide-field surveys programmed at radio wavelengths with SKA, and at optical wavelengths with, e.g., the LSST or similar telescopes. Obviously, the most interesting cases will likely be subject of targeted, monitoring observations with these and other facilities. For example, Gal-Yam et al. (2006) clearly showed the impact of carrying radio and optical follow-up observations of all possible radio transients discovered in surveys covering a 
significant fraction of the sky area, in terms of GRBs and SNe studies.

\section{Radio emission from CCSNe}

In this section we give account of the radio emitting properties, which are crucial to understand the technical issues discussed in the remaining sections.

CCSNe result from both single and binary massive star systems, with initial masses $\gtrsim 8$ $\mathrm{M}_{\odot}$, and include a diversity of spectroscopically divided subtypes (Type IIP/L, IIn, IIb, Ib/c; see e.g. Eldridge et al. 2013). Upon gravitational collapse of the core, the outer parts of the shell are ejected at very high speeds, $v \approx 10000\left(E_{\mathrm{in}} / 10^{51} \mathrm{erg}\right)^{1 / 2}\left(M_{\mathrm{ej}} / 1 \mathrm{M}_{\odot}\right)^{-1 / 2} \mathrm{~km} \mathrm{~s}^{-1}$, and the interaction with the outer, much less dense, material of the progenitor star, drives a blastwave that propagates at speeds as high as $v_{s} \simeq(0.1-0.3) c$ (e.g., Matzner \& McKee 1999). This high-speed shock heats the circumstellar material to temperatures of $\sim 10^{9} \mathrm{~K}$. In addition, a reverse shock propagates back into the stellar envelope at speeds of $500-1000 \mathrm{~km} \mathrm{~s}^{-1}$ relative to the expanding ejecta.

When the supernova shock-wave ploughs through the circumstellar gas, a high-energy density shell forms. Within this shell, electrons are accelerated to relativistic speeds and significant magnetic fields are generated, giving rise to the production of the observed radio emission. The radio emission from supernovae is essentially non-thermal synchrotron emission from relativistic electrons, and is due to circumstellar interaction (e.g., Chevalier 1982). In the framework of the $\mathrm{SN}$-circumstellar interaction scenario, the optically thin radio emission from CCSNe scales with the mass-loss wind parameter, $\mathscr{M}=\dot{M} / v_{w}$, where $\dot{M}$ and $v_{w}$ are the pre-supernova mass-loss rate and wind speed, respectively. Thus, for the radio emission to be appreciable, the progenitor star must have lost a significant amount of mass via a stellar wind and/or stripping due to a close companion. The presupernova stellar wind speed plays an equally important role: the smaller the wind speed, the more mass is retained in the vicinities of the SN progenitor, and the stronger the circumstellar interaction will be. According to their $\mathscr{M}$ value, CCSNe can be divided into two basic groups: Type $\mathrm{Ib} / \mathrm{c} \mathrm{SNe}$, which have expelled fast winds $\left(v_{w} \approx 1000 \mathrm{~km} \mathrm{~s}^{-1}\right)$, have $\mathscr{M} \sim 10^{-8} \mathrm{M}_{\odot} \mathrm{yr}^{-1} / \mathrm{km} \mathrm{s}^{-1}$, thus implying $\dot{M} \sim 10^{-5} \mathrm{M}_{\odot} \mathrm{yr}^{-1}$ during their blue supergiant phase. Type II SNe are characterized by significantly slower $\left(v_{w} \approx 10 \mathrm{~km} \mathrm{~s}^{-1}\right)$ winds, although the large differences in mass-loss rates $\dot{M} \sim\left(10^{-4}-10^{-6}\right) \mathrm{M}_{\odot} \mathrm{yr}^{-1}$ result in a large scatter in $\mathscr{M} \sim\left(10^{-5}-10^{-7}\right) \mathrm{M}_{\odot} \mathrm{yr}^{-1} / \mathrm{km} \mathrm{s}^{-1}$ during their red supergiant phase, which affects the time at which they reach their peak radio luminosity.

This pre-supernova wind, made of thermal electrons, has a power-law density profile, which for a steady, spherically symmetric wind has the following form: $\rho_{\mathrm{CSM}} \propto r^{-2}$. This circumstellar wind is the main responsible for the partial suppression of synchrotron radio emission from supernovae (e.g., Chevalier 1982), so that the radio flux density evolution of many CCSNe can be well described by the following equation (e.g., Weiler et al. 2002)

$$
S_{v}(t)=K_{1}\left(\frac{v}{5 \mathrm{GHz}}\right)^{\alpha}\left(\frac{t-t_{0}}{1 \text { day }}\right)^{\beta} e^{-\tau_{v}},
$$

where $\tau_{v}$ is the optical depth due to external absorption, which for the sake of simplicity is taken to be due only to the (thermal) electrons of the pre-supernova wind and of a possible distant H II region. The optical depth can then be written as 
Table 1: Radio parameters of core-collapse supernovae

\begin{tabular}{lrcccrr}
\hline SN Type & $f_{\text {CCSN }}$ & $\alpha$ & $\beta$ & $\delta$ & $t_{\text {peak (days) }} \times(v / 5 \mathrm{GHz})$ & $L_{\nu, \text { peak }} /\left(10^{27} \mathrm{erg} \mathrm{s}^{-1} \mathrm{~Hz}^{-1}\right)$ \\
\hline Ib/c & $26.0 \%$ & -1.1 & -1.4 & -2.5 & $2-100$ & $0.02-20$ \\
IIb & $12.1 \%$ & -1.1 & -1.0 & -2.0 & 180 & $2-5$ \\
IIP & $55.5 \%$ & -0.7 & $-(0.7-1.2)$ & -3.0 & $30-500$ & $0.2-1$ \\
IIL & $3.0 \%$ & -0.7 & -0.8 & $-(2.7-3.0)$ & $100-800$ & $1-30$ \\
IIn & $2.4 \%$ & -0.7 & $-(1.3-1.7)$ & -3.0 & $\approx 800$ & $10-20$ \\
87A-like & $1.0 \%$ & -1.0 & $\ldots$ & -3.0 & & \\
\hline
\end{tabular}

$$
\tau_{v}=\left(\frac{v}{5 \mathrm{GHz}}\right)^{-2.1}\left[K_{2}\left(\frac{t-t_{0}}{1 \text { day }}\right)^{\delta}+K_{3}\right],
$$

where the first term accounts for the time varying external absorption, which continuously decreases (since $\rho_{\mathrm{CSM}} \propto r^{-2}$ ), and the second term allows for a non-varying absorber, i.e., a distant $\mathrm{H}$ II region. The former term is usually the one that dominates in most CCSNe, but cases where the latter term dominates also exist, e.g., SN 2000ft in NGC 7469 (Alberdi et al. 2006; Pérez-Torres et al. 2009b), or some of the SNe in Arp 299A (Pérez-Torres et al. 2009a, 2010). (Synchrotron selfabsorption seems to be the dominant mechanism in many Type Ib/c SNe; however, this does not affect the discussion below, so for the sake of simplicity we only consider here external absorbers.)

In Table 1 we present typical values for the relevant radio emission parameters in Eqs. 2.1 and 2.2, based on those published in Weiler et al. (2002) and Romero-Cañizales et al. (2014) for a sample of CCSNe. Stripped-envelope SNe (i.e., Type Ib/c and, to some extent, their cousins, Type IIb SNe) have an optically thin spectral index, $\alpha\left(S_{v} \propto v^{\alpha}\right)$, which is steeper than the spectral index of Type II SNe. The combination of high blastwave speed and stripped envelope in Type Ib/c events (and also in Type IIbc supernovae) explains their very short times to peak and their swift radio evolution (steep values of $\beta$ ), compared to the rest of CCSNe.

The tabulated fraction of each CCSN type is taken from Eldridge et al. (2013), which used CCSNe discovered between 1998.00 and 2012.25 (14.25yr) in galaxies with recessional velocities less than $2000 \mathrm{~km} \mathrm{~s}^{-1}$. Type Ibc SNe make up 26\% of all CCSNe in the local universe. $\sim 57 \%$ of all CCSNe are faint radio SNe, i.e., the Type IIP SNe and the peculiar Type II SNe, e.g., SN 1987A; $15 \%$ are the moderately bright Type IIb and Type IIL SNe, and only $\lesssim 3 \%$ are type IIn $\mathrm{SNe}$, the brightest radio events (see Table 1 and Figure 2).

Type IIn SNe show peak luminosities $L_{\text {peak }} \gtrsim(1-2) \times 10^{28} \mathrm{erg} \mathrm{s}^{-1} \mathrm{~Hz}^{-1}$, while Type Ib/c and Type IIb SNe peak at values of a few times $10^{27} \mathrm{erg} \mathrm{s}^{-1} \mathrm{~Hz}^{-1}$. Together, these bright CCSNe account for less than $30 \%$ of all radio supernovae. Most of the remaining $\sim 70 \%$ are type IIP $\mathrm{SNe}$, which are significantly fainter radio supernovae, with peak luminosities $\lesssim$ few times $10^{26}$ erg s ${ }^{-1} \mathrm{~Hz}^{-1}$ (see Fig. 2).

\section{CCSN searches with the SKA}

In this section, we discuss in detail the advantages of a commensal, wide-field, transient survey with the SKA with respect to currently envisioned efforts with other facilities. 


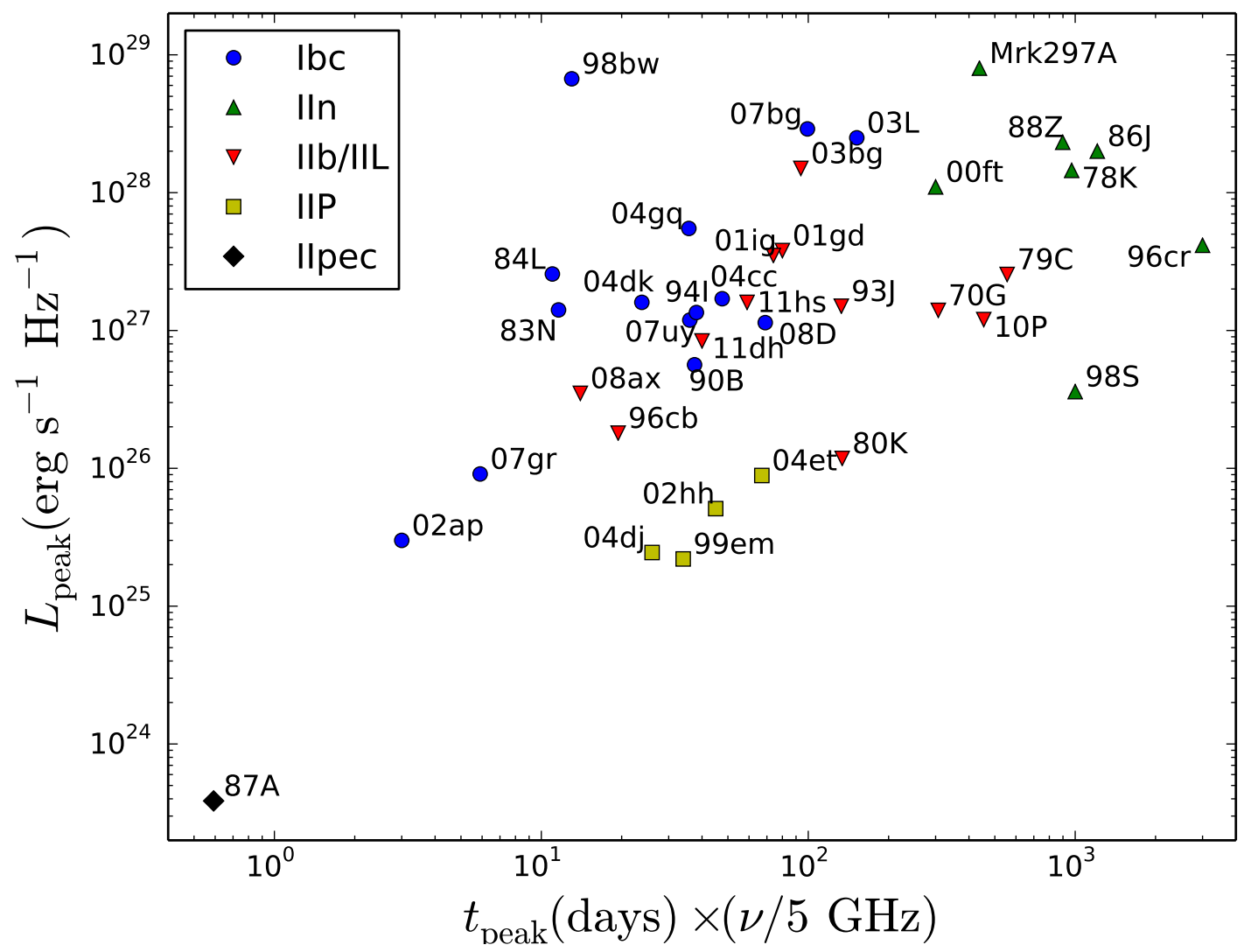

Figure 2: Peak spectral radio luminosity of CCSNe vs. time to peak, times the observing frequency. There is a clear correlation between time to the peak and the luminosity at the peak; in this parameter space, each subtype of CCSNe are located in specific areas; CCSNe show typical peak radio luminosities above $10^{26} \mathrm{erg} \mathrm{s}^{-1} \mathrm{~Hz}^{-1}$, except Type IIP SNe, which usually peak at a few times $10^{25} \mathrm{erg} \mathrm{s}^{-1} \mathrm{~Hz}^{-1}$, and the very rare 87A-like events. Figure adapted from Chevalier et al. (2006) and Romero-Cañizales et al. (2014).

In fact, several wide-field sky surveys will be carried out with the SKA pathfinders MeerKAT and ASKAP, as well as with the upgraded Very Large Array. Those surveys can be used commensally for transient studies, by profitting from programmed wide-field observations. For example, the planned Very Large Array Sky Survey (VLASS) is contemplating the possibility of observing wide field areas (a few hundreds to about one thousand of square degrees) with nominal sensitivities of $1 \sigma \simeq 100 \mu \mathrm{Jy} /$ beam per epoch, aiming at reaching r.m.s. values of $\simeq(40-70) \mu \mathrm{Jy} / \mathrm{beam}$ after stacking multi-epoch observations. However, those sensitivities are just too shallow to be of any real use for CCSN studies. Indeed, a $5 \sigma$ figure of merit corresponds to $500 \mu \mathrm{Jy} / \mathrm{beam}$ per epoch, so that the maximum distance to detect a type IIP event would be $\sim 9$ Mpc (see Table 3). Unless sky areas close to the full celestial sphere are surveyed-which is very unlikely- VLASS will only pick up a handful of CCSNe after one year of observations (see Sect. 3.1 below). Most likely, this handful of CCSNe will be discovered first by optical searches, and some of them will 
Table 2: Relevant observational parameters for the proposed SKA1 designs and comparable telescopes

\begin{tabular}{lccccccc}
\hline & eMERLIN & VLA $^{\dagger}$ & MeerKAT & ASKAP & SKA1-SUR & SKA1-LOW & SKA1-MID \\
\hline FoV $\left(\right.$ deg $\left.^{2}\right)$ & 0.25 & 0.25 & 0.86 & 30 & 18 & 27 & 0.49 \\
Fiducial Freq. $(\mathrm{GHz})$ & 1.4 & 1.4 & 1.4 & 1.4 & 1.67 & 0.11 & 1.67 \\
Resolution $(\operatorname{arcsec})$ & 0.15 & 1.4 & 11 & 7 & 0.9 & 11 & 0.22 \\
Baseline/size $(\mathrm{km})$ & 220 & 35 & 4 & 6 & 50 & 50 & 200 \\
Bandwidth $(\mathrm{MHz})$ & 400 & 1000 & 1000 & 300 & 500 & 250 & 770 \\
Sensitivity $\left(\mu \mathrm{Jy}^{-\mathrm{hr}^{-1 / 2}}\right)$ & 27.1 & 3.9 & 3.2 & 28.9 & 3.7 & 2.1 & 0.7 \\
\hline
\end{tabular}

The VLASS $1-\sigma$ figure is $\gtrsim 10$ times worse than obtained with one hr of VLA observing time.

be subject of targeted radio observations anyway, given their vicinity. Thus, deeper sensitivies are needed to make a substantial contribution to the field.

\subsection{SKA survey strategy for commensal CCSN searches}

The best strategy for transient studies with the SKA is one that combines good angular resolution ( $\lesssim 1.5 \mathrm{arc} \mathrm{sec}$ ) and large field of view, $\left(\mathrm{FoV} \gtrsim 10 \mathrm{deg}^{2}\right)$, at frequencies around and above $\sim 1.5$ GHz. The SKA1-SUR, which should provide an approximate survey sensitivity of $\lesssim 4.2 \mu \mathrm{Jy} / \mathrm{beam}$ after 1-hr of on-source integration (assuming a bandwidth of $500 \mathrm{MHz}$ ) for a FoV of $\simeq 18 \mathrm{deg}^{2}$, with an angular resolution of $\sim 0.9$ arc sec at a fiducial frequency of $1.7 \mathrm{GHz}$, meets such requirements (see Table 2).

SKA1-SUR is likely to observe the sky for $\gtrsim 1000 \mathrm{hr}$ in its first year of operations. Thus, depending on the specific observing band (or bands), the surveyed area will lie in the range of $\sim 9000-18000 \mathrm{deg}^{2}$. As we explain below, to be of use for CCSNe searches, each field of view should be visited five times (each time for an on-source time of 12 minutes), at a cadence of one visit every $\lesssim 90 \mathrm{~d}$. The r.m.s. attained per pointing will be of $9.3 \mu \mathrm{Jy} / \mathrm{beam}$ per pointing, and the r.m.s. for stacked images will be $4.2 \mu \mathrm{Jy} / \mathrm{beam}$, according to the SKA1-SUR specifications. Such a commensal strategy is likely to result in the largest sample of radio supernovae ever detected in the nearby universe.

\subsection{Expectations for CCSN discoveries with wide surveys}

Table 3 summarizes the expectations for detecting CCSNe using the VLASS, SKA1-SUR, SKA-50\% and SKA. More specifically, we show the maximum distance to which a CCSN is expected to be detected above $5 \sigma$, for the nominal r.m.s. values of VLASS $(\sim 100 \mu \mathrm{Jy} / \mathrm{beam} /$ pointing $)$ and SKA1-SUR $(\sim 9.3 \mu \mathrm{Jy} /$ beam/pointing), as well as for SKA1-50\% (i.e., only half of the bandwidth of SKA1-SUR will be available), and SKA (ten times more sensitive than SKA1). Here, we took the approximate median value of the peak luminosity of each CCSN type. In practice, this means that only half of the quoted values in Table 3 will be detected. The other half would lie below our $5 \sigma$ cut and are expected to be missed. In its currently assumed specifications, VLASS will detect Type $\mathrm{Ib} / \mathrm{c} \mathrm{SNe}$ only up to a distance of $\lesssim 58 \mathrm{Mpc}$, less than half the value quoted in Kamble et al. (2014). SKA1-SUR, on the other hand, will be able to detect Type Ib/c SNe up to $\sim 188$ Mpc, i.e., a volume $\sim 35$ times larger than the volume where the VLASS will be sensitive to Type Ib/c SNe. 
For an all-sky survey, the total number of detections in one year can be written as $N_{\text {all-sky }}=$ $\Re \times V \times \min \left[\Delta \mathrm{t}_{\text {bright }} / \Delta \mathrm{t}_{\text {cadence }}, 1\right]$, where $\Re$ is the volumetric rate of CCSNe, $\Delta t_{\text {bright }}$ is the time that a CCSN of a given type remains bright above the survey sensitivity, and $\Delta t_{\text {cadence }}$ is the cadence time of the observations, respectively. As long as $\Delta t_{\text {bright }} \gtrsim \Delta t_{\text {cadence }}$, one can be sure that all CSSNe are detected. Since $\Delta t_{\text {bright }} \simeq \Delta t_{\text {peak }} v_{5}^{-1}$ days (see col. 6 in Table 3), then $\Delta t_{\text {cadence }} \lesssim \Delta t_{\text {peak }} v_{5}^{-1}$ days, where $v_{5}=v / 5 \mathrm{GHz}$. The volumetric rate of CCSNe in the local universe $(z \lesssim 0.1)$ is $\Re \sim 10^{-4}$ $\mathrm{SNe} \mathrm{yr}^{-1} \mathrm{Mpc}^{-3}$ within a factor of $\sim 2$, while at $z \gtrsim 1.0$ the volumetric CCSN rate is $\Re \sim 10^{-3} \mathrm{SNe}$ $\mathrm{yr}^{-1} \mathrm{Mpc}^{-3}$ (see Dahlen et al. 2012 and the right panel of Fig. 1). The total number of CCSNe expected to be detected in one year over the whole sky, in the local universe $(z \lesssim 0.1)$, where $\Re \sim 10^{-4} \mathrm{SNe} \mathrm{y^{-1 }} \mathrm{Mpc}^{-3}$ is then $N_{\text {all-sky }} \approx 420\left(D_{\max } / 100 \mathrm{Mpc}\right)^{3}\left(\Re / 10^{-4} \mathrm{SNe} \mathrm{yr}^{-1} \mathrm{Mpc}^{-3}\right)$, and the expected number of detections of each CCSN type is $f_{\text {CCSN }} \times N_{\text {all-sky }}$, where $f_{\text {CCSN }}$ is the fraction of each CCSN type (see Table 1). The cadence time is set up by the need to detect the fast-evolving type Ib/c SNe. For a fiducial frequency of $1.7 \mathrm{GHz}$, the cadence time should thus be no more than $\sim 90$ days. Note the huge difference in the number of expected CCSNe to be detected by the VLASS and by SKA1-SUR, which is due to the VLASS being $\simeq 10.8$ times less sensitive than SKA1-SUR. Since $N_{\text {det }} \propto D_{\max }^{3} \propto S_{v}^{-3 / 2}$, the expected number of detections in SKA1-SUR is $\simeq 35.3$ times larger than for the VLASS.

We used the above expression for $N_{\text {all-sky }}$, and combined it with the fraction of SNe from each type (second column in Table 1) to obtain the expected number of detected CCSNe in one year, for a survey covering 10,000 $\mathrm{deg}^{2}$ (see Table 3). We also took into account the limiting detectable volumes for each CCSN type, according to their approximated median luminosities. The main limitation is due to the relatively small value of the maximum distance that will allow a detection of a type IIP SN, which is why so few detections of them are expected. (Note that the VLASS will need to sample a $10,000 \mathrm{deg}^{2}$ area for 25 years to blindly discover just one single type IIP event.) On the contrary, the extremely bright Type IIn supernovae would be detectable, in principle, up to a distance of $\sim 422 \mathrm{Mpc}(z \sim 0.1)$ with SKA1, and up to $\sim 1350 \mathrm{Mpc}(z \sim 0.25)$ with SKA, assuming a tenfold increment in sensitivity (see Table 3 ). The relatively high luminosity of spirals at those frequencies $\left(\sim 7 \times 10^{27} \mathrm{erg} \mathrm{s}^{-1} \mathrm{~Hz}^{-1}\right)$ may prevent unambiguous detection of even Type IIn at those distances with SKA1, as the synthesized beam of $1.0^{\prime \prime}$ corresponds to $2.1 \mathrm{kpc}$, which could pick up a significant amount of the galaxy luminosity, thus the need for high angular resolution. This limitation will be overcome once SKA is completed, as is foreseen to have a twentyfold better angular resolution. In addition, one has to take into account that around $10 \%$ of the massive starformation already at $z \sim 0.1$ will come from Luminous Infrared Galaxies (Magnelli et al. 2011). While those galaxies are prolific CCSN factories, their detection with SKA1, or even with SKA, will not be possible in general, as the compact starburst (size $\lesssim 500$ pc) will have a typical 1.4 $\mathrm{GHz}$ brightness well in excess of our 5- $\sigma$ sensitivity limit. For the sake of simplicity, and to stay on the conservative side, we assume here that we recover all Type IIn SNe up to an effective distance of $250 \mathrm{Mpc}$ with SKA1-SUR and SKA1-50\% (see Table 3).

We advocate for SKA1-SUR transient observations at $1.7 \mathrm{GHz}$, which should provide a survey speed of $\simeq 18 \mathrm{deg}^{2} / \mathrm{hr}$. Thus, $10,000 \mathrm{deg}^{2}$ can be covered in $556 \mathrm{hr}$, which easily accomodates into surveys like SKA1-SUR. Indeed, the only requirement for such a programme to be successful under SKA1-SUR is that a minimum number of visits are done to the same field to secure the discovery of recently exploded CCSNe by means of their variability. The cadence time would be limited by 
Table 3: Expectations for CCSN detections in the local Universe from commensal radio surveys from the $\operatorname{VLASS}(5-\sigma=500 \mu \mathrm{Jy} / \mathrm{beam}), \operatorname{SKA} 1-\mathrm{SUR}(5-\sigma=46.5 \mu \mathrm{Jy} / \mathrm{beam}), \mathrm{SKA} 1-50 \%(5-\sigma=66.4 \mu \mathrm{Jy} / \mathrm{beam})$, and SKA $(5-\sigma=4.65 \mu \mathrm{Jy} / \mathrm{beam})$ assuming each survey observes at a nominal frequency of $1.7 \mathrm{GHz}$ and covers an area of $10,000 \mathrm{deg}^{2}$ in one year. $L_{v, 26}=L_{v, \text { peak }} / 10^{26} \mathrm{erg} / \mathrm{s} / \mathrm{Hz} ; v_{5}^{-1}=v / 5 \mathrm{GHz}$

\begin{tabular}{lccrlrlrlrr}
\hline SN Type & $\Delta t_{\text {peak }} v_{5}^{-1}$ & $L_{v, 26}$ & \multicolumn{2}{c}{ VLASS } & \multicolumn{2}{c}{ SKA1-SUR } & \multicolumn{2}{c}{ SKA1-50\% } & \multicolumn{2}{c}{ SKA } \\
\cline { 5 - 10 } & days] & & $D_{\max }$ & $N_{\text {det }}$ & $D_{\max }$ & $N_{\text {det }}$ & $D_{\max }$ & $N_{\text {det }}$ & $D_{\max }$ & $N_{\text {det }}$ \\
\hline Ib/c & 30 & 20 & 58 & 5.1 & 189 & 177 & 159 & 106 & 596 & 5618 \\
IIb, IIL & $\sim 150$ & 10 & 41 & 0.8 & 133 & 29 & 112.2 & 17.4 & 422 & 924 \\
IIP & 40 & 0.5 & 9 & 0.04 & 30 & 1.5 & 25 & 0.9 & 94 & 47 \\
IIn & 1000 & 100 & 129 & 6.6 & 422 & 104 & 355 & 80 & 1334 & 7247 \\
87A & 2 & 0.04 & 2.6 & $\sim 10^{-5}$ & 8.4 & $\sim 10^{-3}$ & 9 & $\sim 10^{-3}$ & 26.7 & 0.05 \\
\hline Total & & & & $\sim 13$ & & $\sim 311$ & & $\sim 204$ & $\sim 13800$ \\
\hline
\end{tabular}

peculiar Type II SNe, e.g., SN 1987A, peaking very early after the explosion, but since we will miss them anyway (see Table 3), the cadence time is actually constrained to $\sim 90$ days by the type Ib/c SNe turnover. This requirement can be easily fulfilled by carrying out five equally spaced 12-min visits of the same field during one year, resulting in an r.m.s. of $9.3 \mu \mathrm{Jy} / \mathrm{beam}$ per visit and thus reaching the specified $4.2 \mu \mathrm{Jy} / \mathrm{beam}$ after five visits (=1 hr on-source).

While the VLASS will detect only $\sim 13$ CCSNe after surveying 10,000 deg ${ }^{2}$ after one year, SKA1-SUR is expected to detect over 300 CCSNe in one year (see Table 3). SKA1-SUR, unlike VLASS, will not need to cover an extremely huge area to ensure a large number of detections. In fact, Table 3 shows that the VLASS needs to cover an area of $\gtrsim 13,000 \mathrm{deg}^{2}$ to detect just one single IIb/IIL event in one year; the detection of a single Type IIP from a blind radio survey within the VLASS, would require about six years of observations of the whole sky (or 25 years of the same $10,000 \mathrm{deg}^{2}$ field). In short, the handful of radio SNe expected to be detected by the VLASS will most likely be detected anyway by nearby optical searches. Clearly, VLASS-class blind radio surveys will not add much to our knowledge of CCSN radio properties and/or statistics. On the contrary, SKA1-SUR, carried out over $\sim 10000 \mathrm{deg}^{2}$ with a cadence time of 90 days, should detect about 177 type Ibc SNe, 29 type IIb/IIL, 1-2 type IIP, and 104 type IIn CCSNe, for a total of $\sim 311$ CCSNe in just one year, or about 26 CCSNe per month, for a total on-source time of 556 hr. Assuming a 33\% overhead time, the total time needed is of $\sim 740 \mathrm{hr}$, or $2.0 \mathrm{hr} /$ day for such a commensal, transient survey to be successful.

There are several reasons for the unrealistic predictions made by Kamble et al. (2014) regarding expectations of CCSN detections within the VLASS. Kamble et al. (2014) mimicked the approach of Lien et al. (2011), who fitted the distribution of radio luminosity peaks of about 20 well observed radio supernovae, concluding that the average peak radio luminosity of all CCSNe was $\gtrsim 10^{27} \mathrm{erg} \mathrm{s}^{-1} \mathrm{~Hz}^{-1}$. However, as Fig. 2 shows, different types of CCSNe peak at significantly different luminosities, so the predictions of both Lien et al. (2011) and Kamble et al. (2014) were too optimistic. Indeed, Kamble et al. (2014) considered all CCSNe to be type $\mathrm{Ib} / \mathrm{c}$, i.e., $f_{\mathrm{Ib} / \mathrm{c}}=f=1.0$, while type $\mathrm{Ib} / \mathrm{c}$ in the local Universe make up about $26 \%\left(f_{\mathrm{Ib} / \mathrm{c}}=0.26\right)$ of all CCSNe. Finally, Kamble et al. (2014) assumed that CCSNe are detected at the $1-\sigma$ level, which clearly cannot be the case. Actually, for a clear detection, a minimum threshold of five times the off-source r.m.s. will be needed, since the number of independent beams within the $1.7 \mathrm{GHz}$ FoV 
will be of several millions with SKA1, and this number will be much larger with the SKA. Therefore, artificial sources above $3 \sigma$ and approaching a $\sim 5 \sigma$ level should be expected, which may result in a substantial fraction of false detections even with a $3 \sigma$ threshold.

When the characteristic radio luminosity of each CCSN type is taken into account, as well as their fraction, and a realistic 5- $\sigma$ detection threshold, numbers get dramatically lower for the intended VLASS in terms of (blind) CCSN radio discoveries. Even for SKA1-SUR, Achille's heel remains the expected low-number statistics for Type IIP SNe, so large areas need to be covered (see Table 3).

\subsection{Impact of a "poor man's" SKA1}

We now quantify the impact of a decrement by a $50 \%$ in the SKA1-SUR specifications (SKA1 $50 \%$ ). If the angular resolution is degraded, this is expected to have a minor effect in terms of CCSN discovery, as most of the SNe in normal, spiral galaxies, will be far from the galaxy nucleus, and therefore should be easily discerned. However, the impact due to a poorer array sensitivity is very relevant. Since $N_{\operatorname{det}} \propto S_{v}^{-3 / 2}$, then a decrement of the available bandwidth by $50 \%$ implies a sensitivity decrement by $\sqrt{2}$, and thus a decrement in the number of CCSN detections of almost $40 \%$, if the initial specifications are not observed.

Although the preferred frequencies are $\gtrsim 1.7 \mathrm{GHz}$, which optimizes FoV, sensitivity, and angular resolution, as well as permits a cadence time significantly larger than at higher frequencies, any of the currently deep, very wide continuum surveys will have a profound, extremely positive impact in the number of CCSN discoveries. In particular, any of the two All-Sky referenced surveys for SKA1 at band 2 will be very beneficial for CCSN searches. Indeed, both surveys propose to image an area of $31000 \mathrm{deg}^{2}$, at $\sim 1.4 \mathrm{GHz}$, reaching r.m.s. sensitivities of 2 (3) $\mu \mathrm{Jy} / \mathrm{beam}$ after two years with an angular resolution of $\sim 0.5(\sim 2)$ arcsec resolution. If any of those surveys is performed, observing the cadence-time of $\sim 90$ days, this would yield increments in the number of CCSNe expected to be detected with respect to the nominal values in Table 3 for SKA1-SUR by factors of 6 and 11, for the All-Sky surveys with $2 \mu \mathrm{Jy} / \mathrm{beam}$ and $3 \mu \mathrm{Jy} / \mathrm{beam}$ sensitivities.

Once SKA is completed, the sensitivity will be about 10 times better than for SKA1, the angular resolution will be about 20 times better, and the FoV around 20 times larger. Therefore, the number of detections is expected to be very close to the number of explosions of all CCSNe in the local universe (see Table 3), and the close to milliarcsecond angular resolution could permit to detect CCSNe even in the dusty environments on (U)LIRGs, thus allowing us to obtain a dust-free, complete census of CCSNe in the local universe. In addition, devoted CCSNe surveys of smaller fields (several degrees to a hundred) with sensitivities of $\sim 100 \mathrm{nJy} / \mathrm{beam}(1 \sigma)$ will be possible with very modest observing times. This will allow us to precisely determine the value and evolution of the fundamental parameter $\Re$ as a function of redshift in the relevant redshift range from $z=0$ to at $z \simeq 1.0$ from radio observations, and compare them with existing estimates (see right panel in Fig. 1).

\section{Type Ia supernovae with the SKA}

Type Ia SNe are the end-products of white dwarfs (WDs) with a mass approaching, or equal to, the Chandrasekhar limit, which results in a thermonuclear explosion of the star. While it is well 
acknowledged that the exploding WD dies in close binary systems, it is still unclear whether the progenitor system is composed of a $\mathrm{C}+\mathrm{O}$ white dwarf and a non-degenerate star (single-degenerate scenario), or both stars are WDs (double-degenerate scenario). In the single-degenerate scenario, a WD accretes mass from a hydrogen-rich companion star before reaching a mass close to the Chandrasekhar mass and going off as supernova, while in the double-degenerate scenario, two WDs merge, with the more-massive WD being thought to tidally disrupt and accrete the lowermass WD (see, e.g., Maoz et al. 2013, and references therein). This lack of knowledge makes it difficult to gain a physical understanding of the explosions, and to model their evolution, thus also compromising their use as distance indicators.

\subsection{Radio and X-ray observations of SNe Ia}

Radio and X-ray observations can potentially discriminate between the progenitor models of $\mathrm{SNe}$ Ia. For example, in all scenarios with mass transfer from a companion, a significant amount of circumstellar gas is expected (see, e.g., Branch et al. 1995), and therefore a shock is bound to form when the supernova ejecta are expelled. The situation would then be very similar to circumstellar interaction in core-collapse $\mathrm{SNe}$ (see above), where the interaction of the blast wave from the supernova with its circumstellar medium results in strong radio and X-ray emission (Chevalier 1982). On the other hand, the double-degenerate scenario will not give rise to any circumstellar medium close to the progenitor system, and hence essentially no prompt radio emission is expected. Nonetheless, we note that the radio emission increases with time in the double-degenerate scenario, contrary to the single-degenerate scenario. This also opens the possibility for confirming the double-degenerate channel in Type Ia SNe via sensitive SKA observations of decades-old Type Ia SNe.

Radio (e.g., Hancock et al. 2011; Panagia et al. 2006) and X-ray (e.g., Hughes et al. 2007; Russell \& Immler 2012) observations of SNe Ia resulted in upper limits on the wind density around SN Ia progenitors of the order of $\dot{M}=1.2 \times 10^{-7} \mathrm{M}_{\odot} \mathrm{yr}^{-1}$, assuming a wind velocity of $10 \mathrm{~km} \mathrm{~s}^{-1}$. At the moment, the deepest radio limits on circumstellar gas come from SNe 2011fe and 2014J. The limits on mass-loss rate from the progenitor system of SN $2011 \mathrm{fe}$ are $\dot{M}=6 \times 10^{-10} \mathrm{M}_{\odot} \mathrm{yr}^{-1}$ and $\dot{M}=2 \times 10^{-9} \mathrm{M}_{\odot} \mathrm{yr}^{-1}$ from radio (Chomiuk et al. 2012) and X-rays (Margutti et al. 2012), respectively, assuming a wind velocity omass-lossf $100 \mathrm{~km} \mathrm{~s}^{-1}$. Similarly, the mass-loss rate limits for the progenitor system of SN 2014J are $\dot{M}=7 \times 10^{-10} \mathrm{M}_{\odot} \mathrm{yr}^{-1}$ and $\dot{M}=1.2 \times 10^{-9} \mathrm{M}_{\odot} \mathrm{yr}^{-1}$ from radio (Pérez-Torres et al. 2014) and X-rays (Margutti et al. 2014), respectively, for a wind velocity of $100 \mathrm{~km} \mathrm{~s}^{-1}$. The above limits permit to rule out all symbiotic systems and the majority of the parameter space associated with stable nuclear burning WDs, as viable progenitor systems for either SN 2011fe or SN 2014J. Recurrent novae with main sequence or subgiant donors cannot be ruled out completely, yet most of their parameter space is also excluded by those radio observations (see Fig. 3.)

\subsection{Unveiling the progenitor scenarios of type Ia SNe with the SKA}

With the advent of the SKA, we will be able to obtain significantly deeper radio limits, or an eventual detection, for SNe Ia exploding at the distance of M 82. For more distant supernovae, we will be able to obtain similar or even more constraining limits to those obtained for SNe $2011 \mathrm{fe}$ 


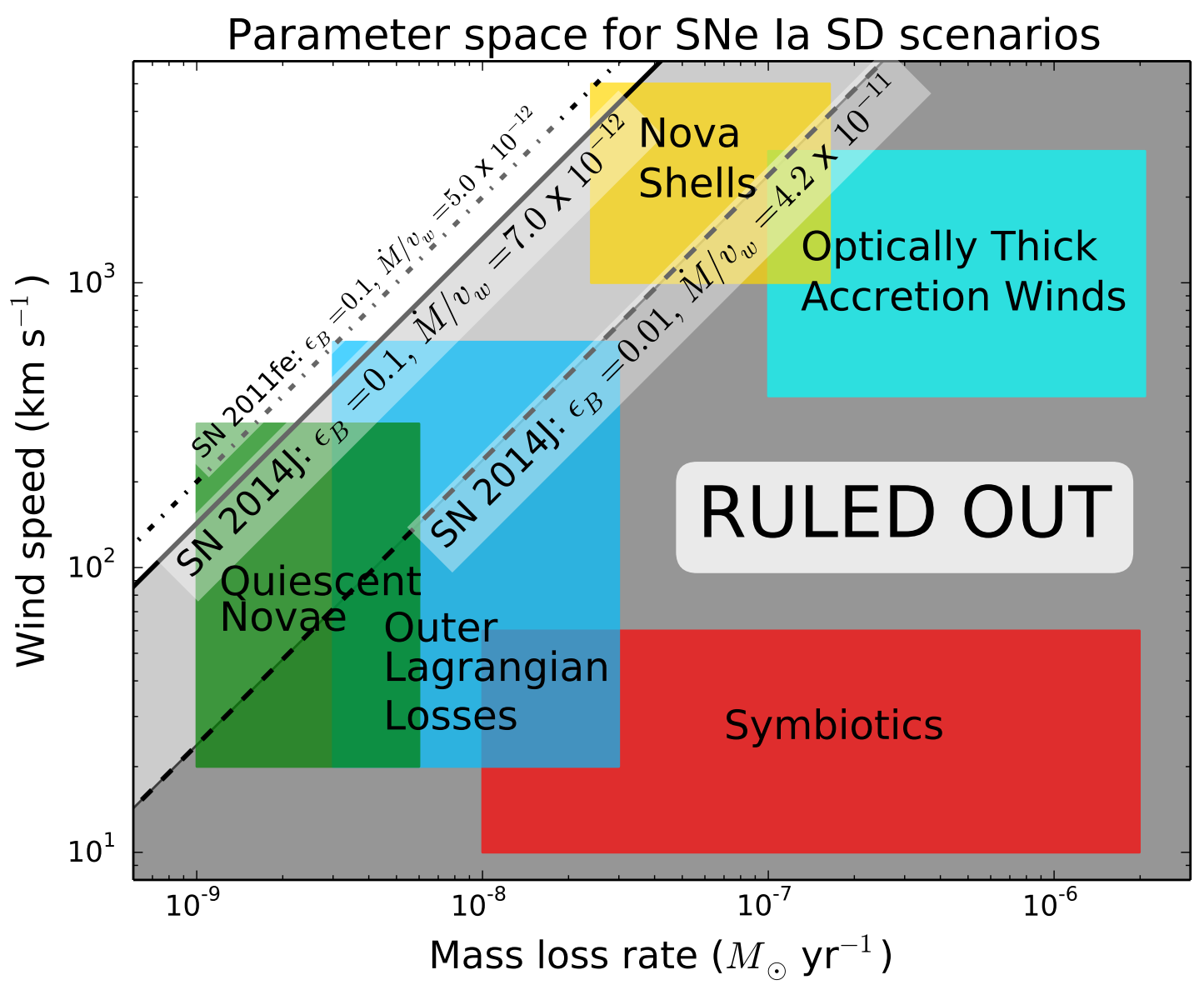

Figure 3: Constraints on the parameter space (wind speed vs. mass-loss rate) for single degenerate scenarios for SN 2014J (see Pérez-Torres et al. 2014 for details). Progenitor scenarios are plotted as schematic zones, following Chomiuk et al. (2012). We indicate our $3 \sigma$ limits on $\dot{M} / \nu_{w}$, assuming $\varepsilon_{\mathrm{B}}=0.1$ (solid line) and the conservative case of $\varepsilon_{\mathrm{B}}=0.01$ (dashed line). Mass loss scenarios falling into the gray-shaded areas should have been detected by the deep radio observations, and therefore are ruled out for SN 2014J. For a comparison, we have included also the limit on SN 2011fe (dash-dotted line) for the same choice of parameters as the solid line for SN 2014J, which essentially leaves only room for quiescent nova emission as a viable alternative among the single-degenerate scenarios for SN 2011 fe (see Pérez-Torres et al. (2014) for details).

and 2014J, which will allow us to build a picture from a larger statistical sample of observed SNe Ia.

The first phase of SKA considers three different surveys: SKA1-LOW, -mid, and -sur (see also Table 2). SKA1-MID, promises to yield $1 \sigma$ sensitivities of $\sim 0.7 \mu \mathrm{Jy} /$ beam in one hour at a fiducial frequency of $1.7 \mathrm{GHz}$. This figure is five times better than currently provided by the most sensitive array, the VLA. SKA1-MID will be able to either detect the putative radio emission of SN 2014J-like objects up to distances $\lesssim 8 \mathrm{Mpc}$ in less than one hour, or put significantly better constraints on the parameter space of single-degenerate scenarios for the next Type Ia SN that explodes at a distance no larger than that to M 82. However, the expected number of SNe Ia per 


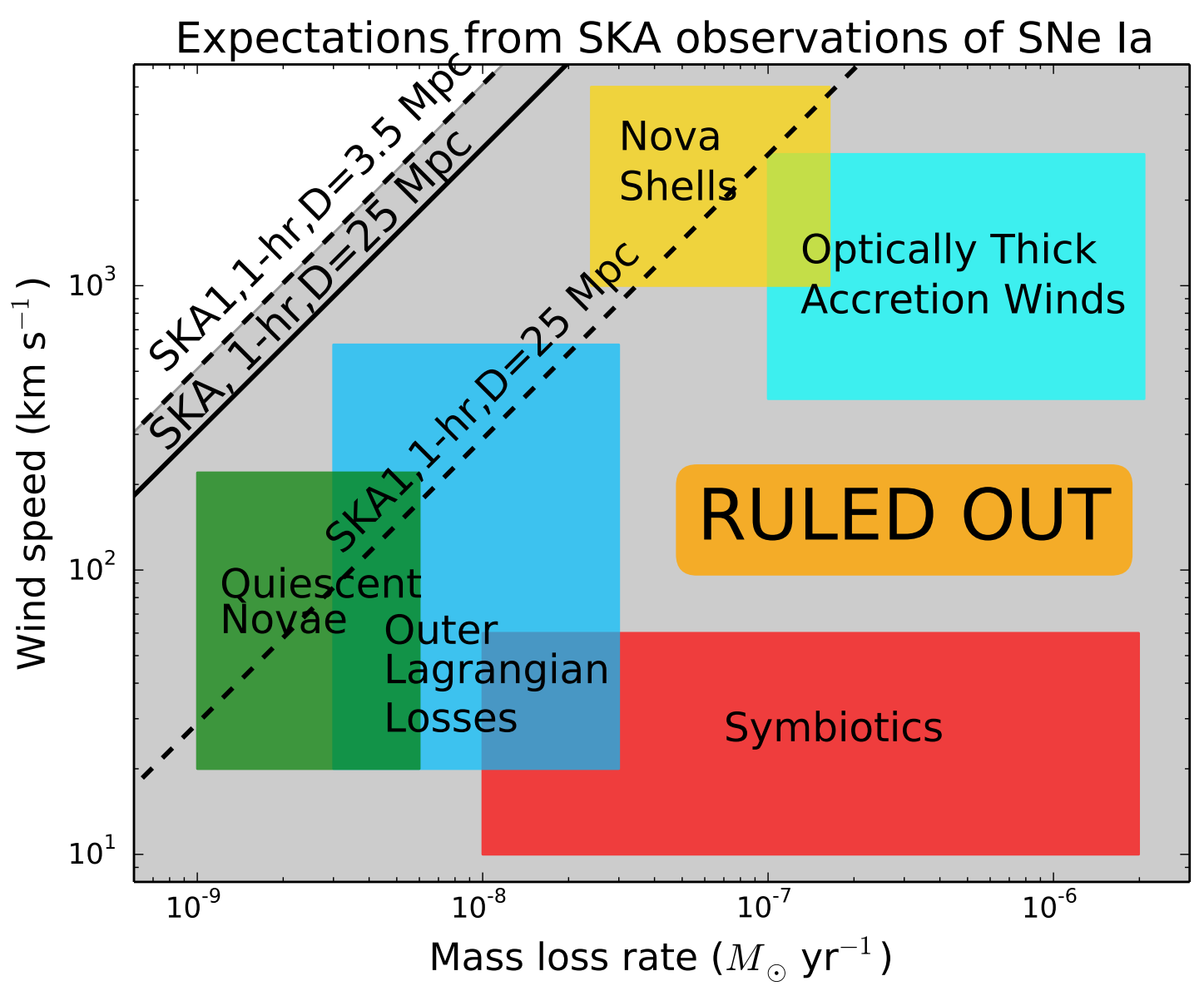

Figure 4: Constraints on the parameter space (wind speed vs. mass-loss rate) for the same single-degenerate scenarios as in Fig. 3, as expected with SKA1 (dashed lines) and the full SKA (solid line). SKA1 will be able to unambiguously probe all single degenerate scenarios for SNe Ia exploding at distances similar to that of $M 82(3.5 \mathrm{Mpc})$, and will be more sensitive than current state of the art, deep radio observations of SN 2014J in M 82, up to a distance of 25 Mpc, or even larger. When SKA is completed, we will be able to unambiguously probe the prompt radio emission within the single degenerate scenario up to distances of $\geq 25 \mathrm{Mpc}$. All lines correspond to 3- $\sigma$.

year in such a volume of the local universe is small. Since the volumetric rate of Type Ia $\mathrm{SNe}$ is $\sim 3 \times 10^{-5} \mathrm{SN} \mathrm{yr}^{-1} \mathrm{Mpc}^{-3}$ (Dilday et al. 2010), we should expect on average one Type Ia SN every $\sim 15 \mathrm{yr}$ within a distance of $\lesssim 8 \mathrm{Mpc}$ (more than twice the distance to M 82 ), which is a depressingly small value to get any statistical improvement in a sensible amount of time. Smartt (2009) found 26 Type Ia SNe out of $132 \mathrm{SNe}$ from a 10.5 year-long survey within $28 \mathrm{Mpc}$. This figure corresponds to about $1 \mathrm{SN}$ Ia every $13 \mathrm{yr}$ within a distance of $8 \mathrm{Mpc}$, in agreement with the value found by Dilday et al. (2010).

To obtain a statistically significant sample of SNe Ia observed in radio and with similar upper limits to those obtained for SNe $2011 \mathrm{fe}$ and $2014 \mathrm{~J}$, we need to sample significantly larger volumes and need much more sensitive radio observations. For example, by sampling out to a distance of 25 Mpc, we can expect $\sim 2$ SNe Ia per year within this sampled volume, which in 10 years would result 
in a total of $\sim 20 \mathrm{SNe}$ Ia, enough to extract statistical results. At this maximum distance, we need a sensitivity $\sim 50$ times better than obtained by the deep radio observations discussed in Chomiuk et al. (2012) and Pérez-Torres et al. (2014), i.e., $80 \mathrm{nJy} /$ beam (1- $\sigma$ ), to be as constraining. The fiducial $1-\sigma$ sensitivity of SKA should be 10 times better than that of SKA1-MID, or about $\sim 70 \mathrm{nJy} / \mathrm{beam}$ in one hour, which will allow to obtain deep radio limits (or eventually the detection) of type Ia $\mathrm{SNe}$ in a short amount of time and out to $25 \mathrm{Mpc}$, or even further. To get a more clear idea of what can be reached with SKA1 and SKA, we plot in Fig. 4 the constraints on the mass-loss rate parameter for an upper limit of $3-\sigma(3 \times 0.7 \mu \mathrm{Jy} /$ beam for SKA1, or $3 \times 70 \mathrm{nJy} /$ beam for SKA) for a Type Ia SN exploding at the distance of M 82 and at $25 \mathrm{Mpc}$. It is evident that, at this level of sensitivity, a non-detection would be essentially as meaningful as a detection, since the former would imply that only the double-degenerate scenario is viable, while the latter would tell us which of the single-degenerate channels results in Type Ia SNe. The overall time needed to carry out such a target-of-opportunity programme will require no more than about $12-24 \mathrm{hr} / \mathrm{year}$, overheads included, for an average of two targets/year within a radius of $25 \mathrm{Mpc}$. Such modest time requests can be easily accommodated within a sensible period of time.

\section{Summary}

We have presented the prospects for advancing our understanding of the physics of supernovae via their study at radio wavelengths with the SKA. Our suggested approach for core-collapse supernovae is a commensal one, taking advantage of the deep, sensitive surveys that are planned with SKA1. We have discussed the expectations for CCSN studies under the specific case of SKA1SUR $\left(\sim 1000 \mathrm{hr}\right.$ in one year, rms=4.2 $\mu \mathrm{Jy} /$ beam in $1-\mathrm{hr}, \mathrm{FoV}=18 \mathrm{deg}^{2}$, bandwidth=500 MHz). In particular, we assumed a fiducial frequency of $1.7 \mathrm{GHz}$ and a covered area of $10000 \mathrm{deg}^{2}$ in one year. The expected number of new CCSNe discovered after one year would be $\gtrsim 310$. For SKA, the expected number of expected CCSN discoveries is well over 10,000 in one year. Therefore, the number of detections is expected to approach the number of explosions of all CCSNe in the local universe, thus allowing us to obtain a dust-free, complete census of CCSNe. The only request from such a programme is a multi-epoch approach, observing a cadence-time of $\lesssim 90 v_{1.7}^{-1}$ days, where $v_{1.7}=v / 1.7 \mathrm{GHz}$. We also note that the proposed programme can be carried out as well at higher frequency bands, as long as an adequate cadence time is observed.

We have also discussed the prospects for probing Type Ia SNe progenitor scenarios with the SKA. The SKA can be used at very low time-cost for searching the putative prompt radio emission arising, in the single-degenerate scenario, from the circumstellar medium around Type Ia SN progenitors in the nearby universe. Complementarily, since the radio emission of Type Ia SNe is expected to increase with time in the double-degenerate scenario, the SKA should observe decadesold, nearby Type Ia SNe, which can potentially confirm the double-degenerate scenario in them. In conclusion, the huge improvement in sensitivity of the SKA with respect to their predecessors should allow us to unambiguously discern which progenitor scenario (single-degenerate vs. double-degenerate) applies to them, thus solving this long-standing issue. 


\section{Acknowledgments}

MAPT, AA, RHI, JMM, and ER acknowledge support from the Spanish Ministry of Economy and Competitiveness (MINECO) through grants AYA2012-38491-C02-01 and AYA201238491-C02-02. PL acknowledges support from the Swedish Research Council. JMM and ER also acknowledge support from the Generalitat Valenciana through grants PROMETEO/2009/104 and PROMETEOII/2014/057. CRC acknowledges financial support from the ALMA-CONICYT FUND Project 31100004. CRC is also supported by the Ministry of Economy, Development, and Tourism's Millennium Science Initiative through grant IC120009, awarded to The Millennium Institute of Astrophysics, MAS.

\section{References}

Alberdi, A., Colina, L., Torrelles, J. M., et al. 2006, ApJ, 638, 938

Batejat, F., Conway, J. E., Hurley, R., et al. 2011, ApJ, 740, 95

Bietenholz, M. F., De Colle, F., Granot, J., Bartel, N., \& Soderberg, A. M. 2014, MNRAS, 440, 821

Bondi, M., Pérez-Torres, M. A., Herrero-Illana, R., \& Alberdi, A. 2012, A\&A, 539, A134

Branch, D., Livio, M., Yungelson, L. R., Boffi, F. R., \& Baron, E. 1995, PASP, 107, 1019

Chevalier, R. A. 1982, ApJ, 259, 302

Chevalier, R. A. 1998, ApJ, 499, 810

Chevalier, R. A., Fransson, C., \& Nymark, T. K. 2006, ApJ, 641, 1029

Chomiuk, L., Soderberg, A. M., Moe, M., et al. 2012, ApJ, 750, 164

Colina, L., Alberdi, A., Torrelles, J. M., Panagia, N., \& Wilson, A. S. 2001, ApJ, 553, L19

Dahlen, T., Strolger, L.-G., Riess, A. G., et al. 2012, ApJ, 757, 70

Dilday, B., Smith, M., Bassett, B., et al. 2010, ApJ, 713, 1026

Eldridge, J. J., Fraser, M., Smartt, S. J., Maund, J. R., \& Crockett, R. M. 2013, MNRAS, 436, 774

Gal-Yam, A., Ofek, E. O., Poznanski, D., et al. 2006, ApJ, 639, 331

Hancock, P. J., Gaensler, B. M., \& Murphy, T. 2011, ApJ, 735, L35

Horiuchi, S., Beacom, J. F., Kochanek, C. S., et al. 2011, ApJ, 738, 154

Hughes, J. P., Chugai, N., Chevalier, R., Lundqvist, P., \& Schlegel, E. 2007, ApJ, 670, 1260

Kamble, A., Soderberg, A., Berger, E., et al. 2014, arXiv:1401.1221

Kankare, E., Mattila, S., Ryder, S., et al. 2014, MNRAS, 440, 1052

Lien, A., Chakraborty, N., Fields, B. D., \& Kemball, A. 2011, ApJ, 740, 23

Magnelli, B., Elbaz, D., Chary, R. R., et al. 2011, A\&A, 528, A35

Mannucci, F., Della Valle, M., \& Panagia, N. 2007, MNRAS, 377, 1229

Maoz, D., Mannucci, F., \& Nelemans, G. 2013, arXiv:1312.0628

Margutti, R., Soderberg, A. M., Chomiuk, L., et al. 2012, ApJ, 751, 134

Margutti, R., Parrent, J., Kamble, A., et al. 2014, ApJ, 790, 52

Mattila, S., Dahlen, T., Efstathiou, A., et al. 2012, ApJ, 756, 111

Matzner, C. D., \& McKee, C. F. 1999, ApJ, 510, 379

Panagia, N., Van Dyk, S. D., Weiler, K. W., et al. 2006, ApJ, 646, 369

Parra, R., Conway, J. E., Diamond, P. J., et al. 2007, ApJ, 659, 314 
Pérez-Torres, M. A., Alberdi, A., Colina, L., et al. 2009, MNRAS, 399, 1641

Pérez-Torres, M. A., Romero-Cañizales, C., Alberdi, A., \& Polatidis, A. 2009, A\&A, 507, L17

Pérez-Torres, M. A., Alberdi, A., Romero-Cañizales, C., \& Bondi, M. 2010, A\&A, 519, L5

Pérez-Torres, M. A., Lundqvist, P., Beswick, R. J., et al. 2014, ApJ, 792, 38

Romero-Cañizales, C., Mattila, S., Alberdi, A., et al. 2011, MNRAS, 415, 2688

Romero-Cañizales, C., Herrero-Illana, R., Pérez-Torres, M. A., et al. 2014, MNRAS, 440, 1067

Russell, B. R., \& Immler, S. 2012, ApJ, 748, L29

Smartt, S. J. 2009, ARA\&A, 47, 63

Soderberg, A. M., Nakar, E., Berger, E., \& Kulkarni, S. R. 2006, ApJ, 638, 930

Weiler, K. W., Panagia, N., Montes, M. J., \& Sramek, R. A. 2002, ARA\&A, 40, 387 